\title{
FTIR Spectra of Magnetic Charge Transfer Complexes of TEMPO Free Radical
}

\author{
Vishal.R.Jain ${ }^{1}$, Hitesh Parmar $^{2}$, Ketan Dodiya ${ }^{3}$, Sagar.M.Agravat ${ }^{3}$ and A.T.Oza $^{3}$ \\ ${ }^{1}$ Navjevan Science College, Dahod, Gujarat,India. \\ ${ }^{2}$ Arts Science And R.A.Patel Commerce College, Bhadran, Gujarat,India, \\ ${ }^{3}$ Department of Physics,Sardar Patel University,Vallabh Vidyanagar - 388120 Gujarat,India.
}

Abstract: The Fourier - transform infrared spectra of complexes of TEMPO free radical with organic acceptors like TCNE, DDQ, TCNQ, Chloranil has been studied. Some of them have ferro or antiferromagnetic properties at very low temperatures. From FTIR spectra, these complexes are found to be small band-gap semiconductors. They are interpreted as Hubbard semiconductors having non-universal band gap. They also show either asymmetric or symmetric Gaussian band revealing electronic delocalization. They are expected to be paramagnetic.

\section{Introduction :}

TEMPO (2,2,6,6 - tetramethyl piporidinyloxy) radical and its substituted derivaties are found to form CTCS with organic acceptors. These CTCS are magnetic, either ferromagnetic or anti-ferromagnetic, at very low temperatures (1-11). The CTCS of TEMPO radical with TCNQ, TCNE, DDQ, chloranil and iodine are prepared in the present work and studied with FTIR spectroscopy. TEMPO acts as a donor because of electron accepting four methyl groups.

\section{Expermental :}

TEMPO free radical was obtained in powder form from SigmaAldrich chemical company,USA.It was mixed with acceptors such as TCNE (tetracyano - p - ethylene), TCNQ (7,7,8,8 tetracyano - p - quino - dimethane), Chloranil DDQ (2,3 dichloro - 5,6 - dicyno - p - benzoquinone) and iodine also obtained as analytical reagents. After mixing, the mixtures were grinded in an agate mortar with a pastle till the colours changed. These homogeneously fine powders were mixed and grinded with spectrograde dry (anhydrous) $\mathrm{KBr}$ powder. Semitransparent pallets were prepared using a manually operated compressing machine and a die. The circular discs prepared in this manner were placed in the dark chamber of an FTIR spectrophotometer.

The spectra in the range $400-4000 \mathrm{~cm}^{-1}$ recorded using a GXFTIR single beam spectrophotometer which is manufactured by Perkin Elmer, USA. It was having a scan range of 15,000 - 30 $\mathrm{cm}^{-1}$, resolution of $0.15 \mathrm{~cm}^{-1}$, an OPD velocity of $0.20 \mathrm{~cm} / \mathrm{sec}$, a scan time of $20 \mathrm{scan} / \mathrm{sec}$ and FIRTGS and MIRTGS detectors. The spectra were recorded in purge mode. A beam splitter of opt $\mathrm{KBr}$ type was used having a range of $7800-370 \mathrm{~cm}^{-1}$.

\section{Results and Discussion :}

The molecular structure of TEMPO free radical is shown (figure 1). The Fourier - transformed infrared (FTIR) spectrum of TEMPO free radical is shown (figure 2). TEMPO radical itself is an insulator having a transmitting range in IR spectrum. An asymmetric gaussian in the range $1000-1700 \mathrm{~cm}^{-1}$ is observed in this spectrum. It is related with optical properties in small polarons model (12). There is hopping of polarons giving rise to this gaussian band of about $10 \%$ absorption below a transmitting range. Also there is a semicircular distribution, marked as SCD, in the low frequency range and centered about $700 \mathrm{~cm}^{-1}$. This distribution is related with random orientations of TEMPO 
molecules in three dimensions in a crystal. The disorder is isotropic giving rise to a semicircular distribution.

The FTIR spectrum of TEMPO - TCNQ charge transfer complex is shown (figure 3). This spectrum covers a range of nature of transition for absorption function and a range of symmetric gaussian distribution, the latter being marked as G. The absorption A is fitted as $\mathrm{Ah} \gamma=\mathrm{B}(\mathrm{h} \gamma-\mathrm{Eg})$ - a nature of allowed indirect or a forbidden direct transition in two - dimensional conductors (figure 4a). Thus TEMPO - TCNQ is a two dimensional semiconductor having $\mathrm{Eg}=0.325 \mathrm{eV}$. It is a non universal Hubbard semiconductor. The gaussian distribution is also fitted by plotting $\ln \mathrm{A}$ vs $(\mathrm{K}-\mathrm{Ko})^{2}$ (figure $4 \mathrm{~b}$ ). The symmetric gaussian distribution is related with electronic delocalization.

The FTIR spectrum of of TEMPO - TCNQ is shown (figure 5). The range of nature of transition is marked as NT and asymmetric gaussian background is marked as AG. The transition is found to be forbidden indirect type by fitting Ah $\gamma=$ $\mathrm{B}(\mathrm{h} \gamma-\mathrm{Eg})^{3}$ (figure 6a). The asymmetric gaussian is fitted by plotting $(\ln \mathrm{A})^{1 / 2}$ vs $\mathrm{K}-\mathrm{K} 0$ on one side of the gaussian band (figure $6 \mathrm{~b}$ ). Both sides of gaussians have different full - widths at half - maxima. The slopes of $(\ln \mathrm{A})^{1 / 2}$ vs K-Ko lines are different for $\mathrm{K}>\mathrm{Ko}$ and $\mathrm{K}<\mathrm{Ko}$. The asymmetric gaussian band can be explained with optical properties in small polaron model (12). Band gap is about $0.272 \mathrm{eV}$.

The FTIR spectrum of TEMPO - DDQ is shown (figure 7). The spectrum reveals a range of nature of transition and two gaussian bands. The nature of transition shows a forbidden direct type transition fitting $\mathrm{Ah} \gamma=\mathrm{B}(\mathrm{h} \gamma-\mathrm{Eg})^{3}$ with $\mathrm{Eg}=0.225 \mathrm{eV}$. It is a universal Peierls gap. Thus an asymmetric molecule like DDQ rather than symmetir TCNQ and TCNE shows a Peierls gap. Both two - dimensional character with electronic delocalization. The nature of transition and one of the gaussian bands are analyzed (figure $8 \mathrm{a}$ and $8 \mathrm{~b}$ ).

The FTIR spectrum of TEMPO - chloranil is shown (figure 9). It shows a nature of transition and oscillations in the density of states. The transition is found to be allowed direct type by fitting
$\mathrm{Ah} \gamma=\mathrm{B}(\mathrm{h} \gamma-\mathrm{Eg})^{1 / 2}$ with $\mathrm{Eg}=0.25 \mathrm{eV}$ - again a Hubbard gaSp. The direct transition is usually found for small donor and small acceptor in a charge transfer complex (figure 10). Below 1800 $\mathrm{cm}^{-1}$, three repeated structures of square - root singularities along a homomolecular sublattice of chloranil stacks are found. This is observed usually in chloranil complexes.

Finally, the FTIR spectrum of TEMPO - $\mathrm{I}_{2}$ is shown (figure 11). The range of nature of transition and asymmetric gaussian band are fitted (figure 12a and 12b). The transition is allowed direct type by fitting $\mathrm{Ah} \gamma=\mathrm{B}(\mathrm{h} \gamma-\mathrm{Eg})^{1 / 2}$ with $\mathrm{Eg}=0.225 \mathrm{eV}$ again attributed to a universal Peierls gap. Thus asymmetric $\mathrm{I}_{3}{ }^{-}$ion also leads to a Peierls gap. Asymmetric gaussian is fitted by plotting $(\ln \mathrm{A})^{1 / 2}$ vs $\mathrm{K}-\mathrm{Ko}$, on one side of the gaussian band. Other side shows a different slope giving a different full width at half - maximum. This can be explained with optical properties in small polaron model (12). The CTCS of TEMPO radical are found to be ferromagnetic of anti - ferromagnetic at very low temperature (11). However, semiconducting nature having either a Hubbard gap or a Peierls gap found in the present work shows that these CTCS are diamagnetic at room temperature. These small band gap semiconductors can not undergo a transition to a magnetic state without being converted into a Pauli paramagnetic phase. Thus a semiconductor to metal type transition should occur at intermediate temperature. This can happen due to thermal contraction of the semiconductors upon cooling. The intermolecular distance decreases due to thermal contraction at low temperature which can lead to more orbital overlap. Thus $\mathrm{p}-\mathrm{p}$ overlap leads to a transition to metallic state which has Pauli paramagmetism. At still low temperatures these CTCS can become magnetic by increase in exchange interaction. Thermal contraction leads to more exchange interaction at low temperature.

\section{Conclusions :}

The symmetric acceptor molecules such as TCNQ, TCNE and Chloranil gives rise to Hubbard semiconducting nature of CTCS of TEMPO radical while asymmetric molecules like DDQ and $\mathrm{I}_{3}{ }^{-}$ion give rise to Peierls semiconducting nature. Symmetric gaussians and asymmetric gaussans arer related with 

www.ijtsrd.com

electronic delocalization and small Polaron hopping. Direct and paramagnrtic phase with orbital overlap due to thermal indirect transitions are related with one or two - contraction.

dimensionalities of CTCS of TEMPO radical. Magnetic nature

at low temperature can be explained with a transition to aPauli

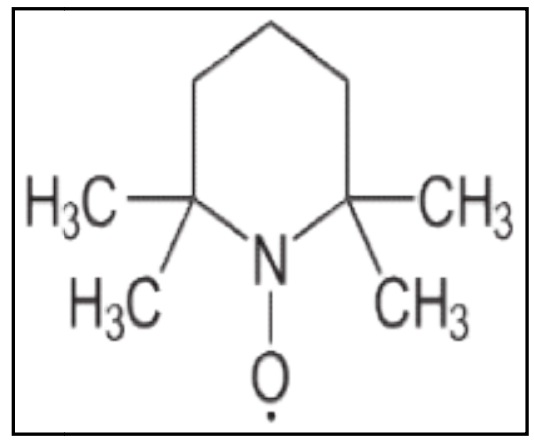

Figure-1: Molecular structure of TEMPO (2,2,6,6-Tetramethylpiperidinyloxyl

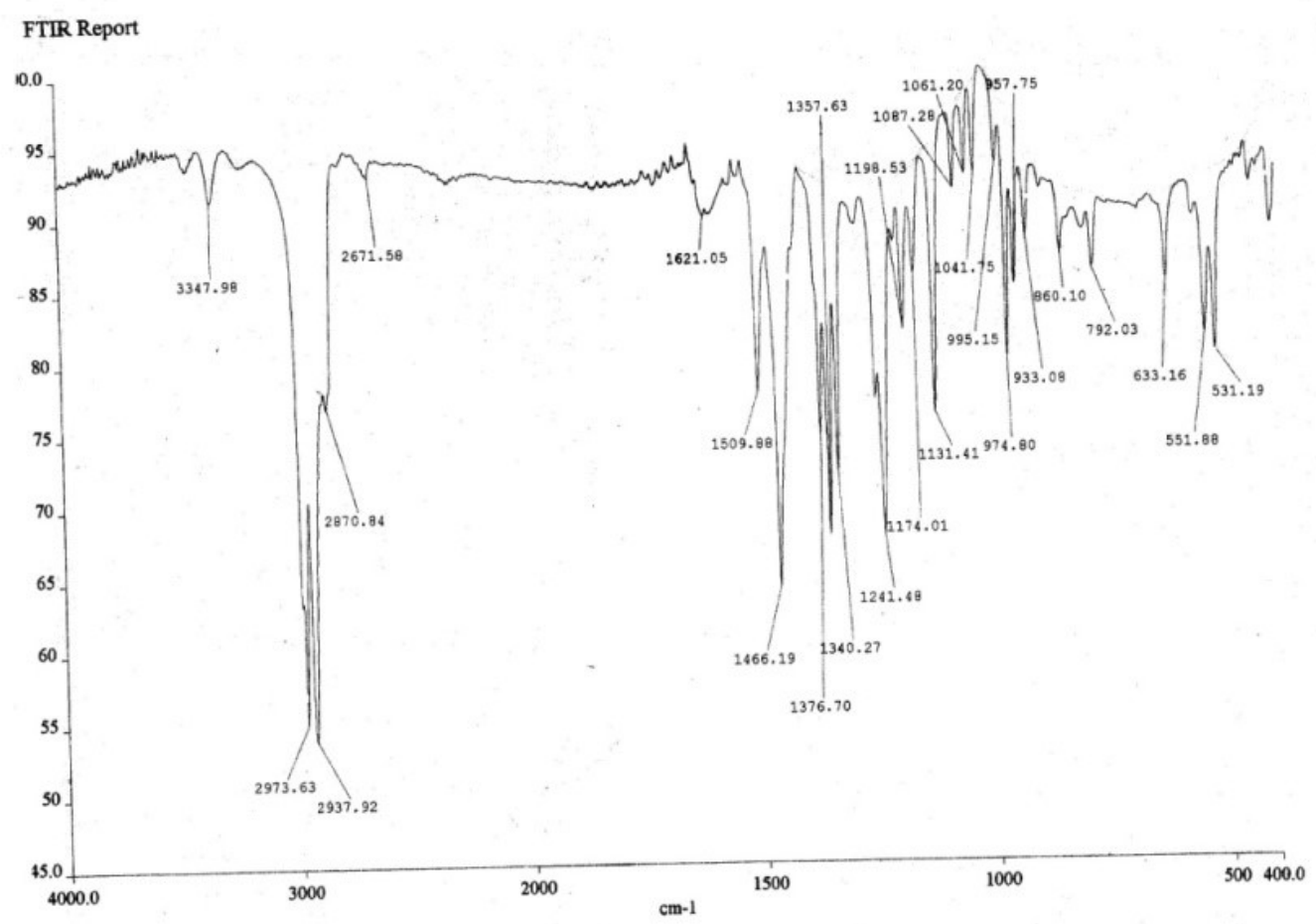

Figure 2 FTIR spectrum of TEMPO 


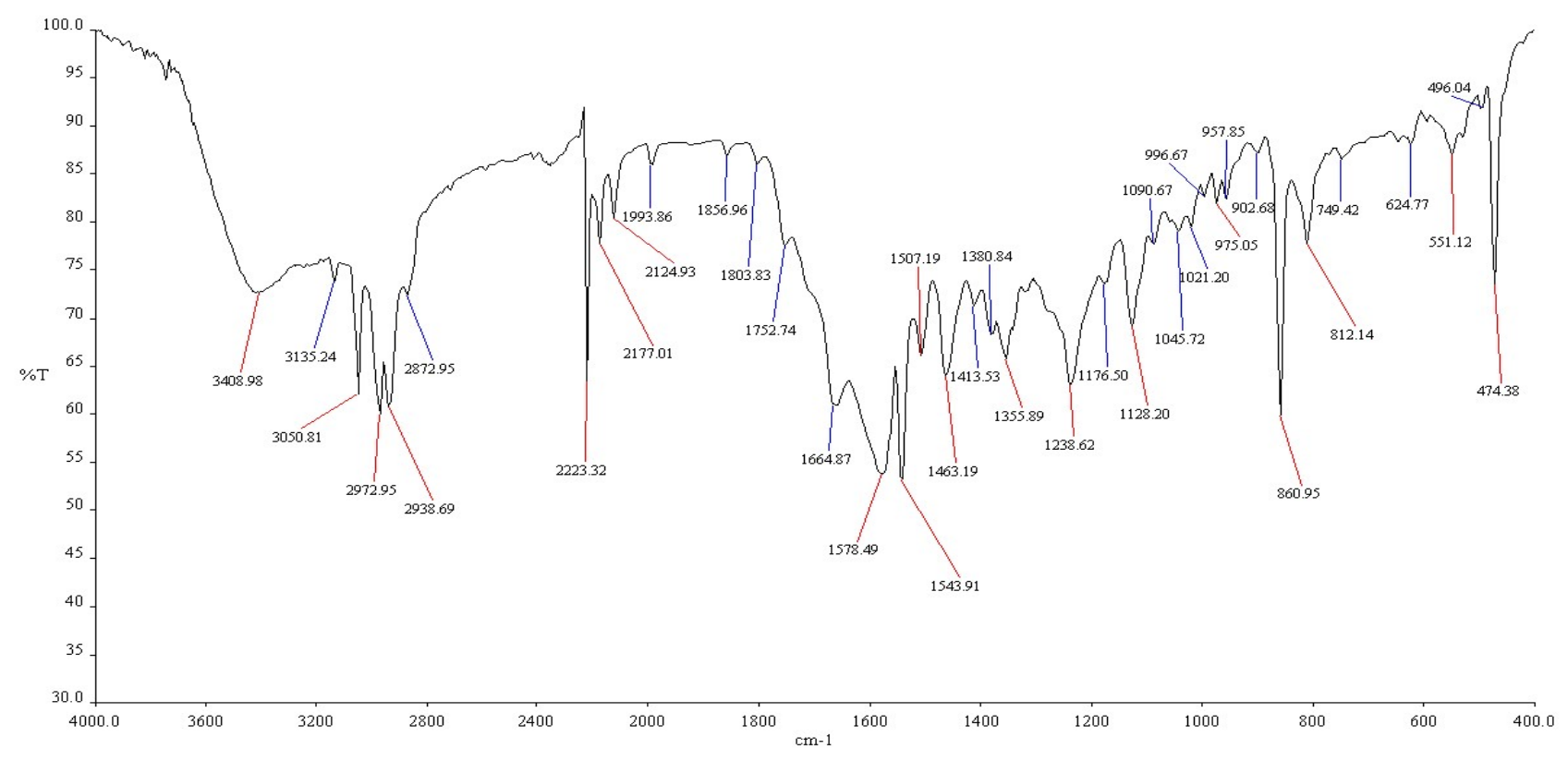

Figure-3 : FTIR spectrum of TEMPO -TCNQ

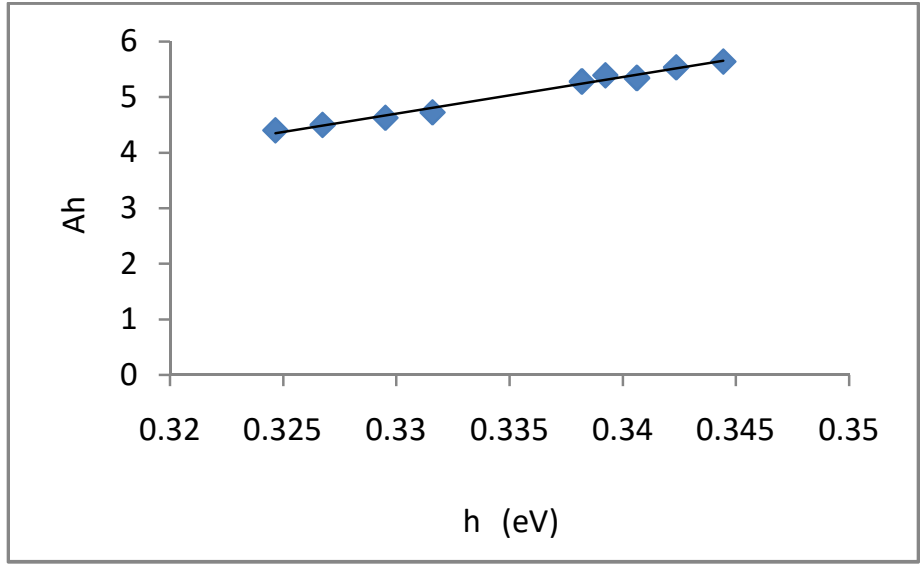

Figure 4a NT of TEMPO -TCNQ

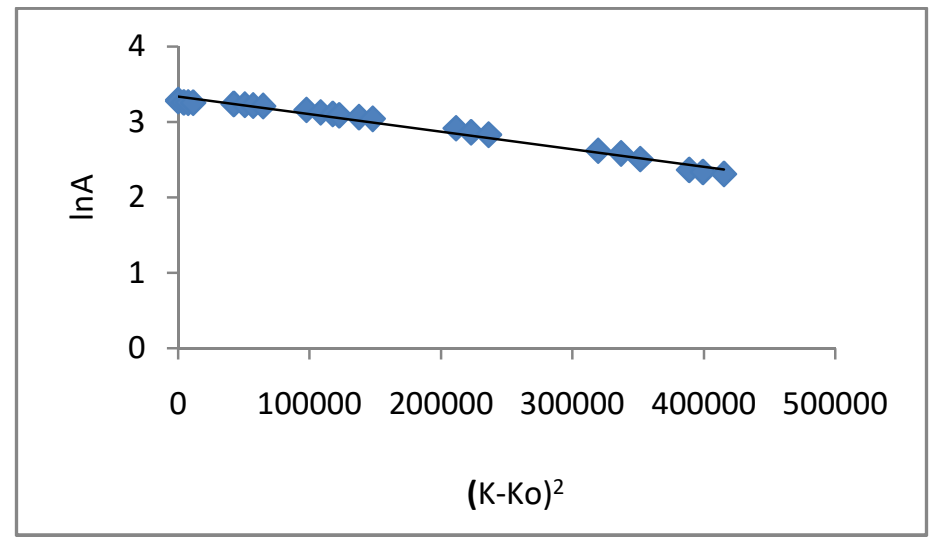

Figure 4b Symmetric Gaussian of TEMPO -TCNQ 
International Journal of Trend in Scientific Research and Development, Volume 1(2), ISSN: 2456-6470 www.ijtsrd.com

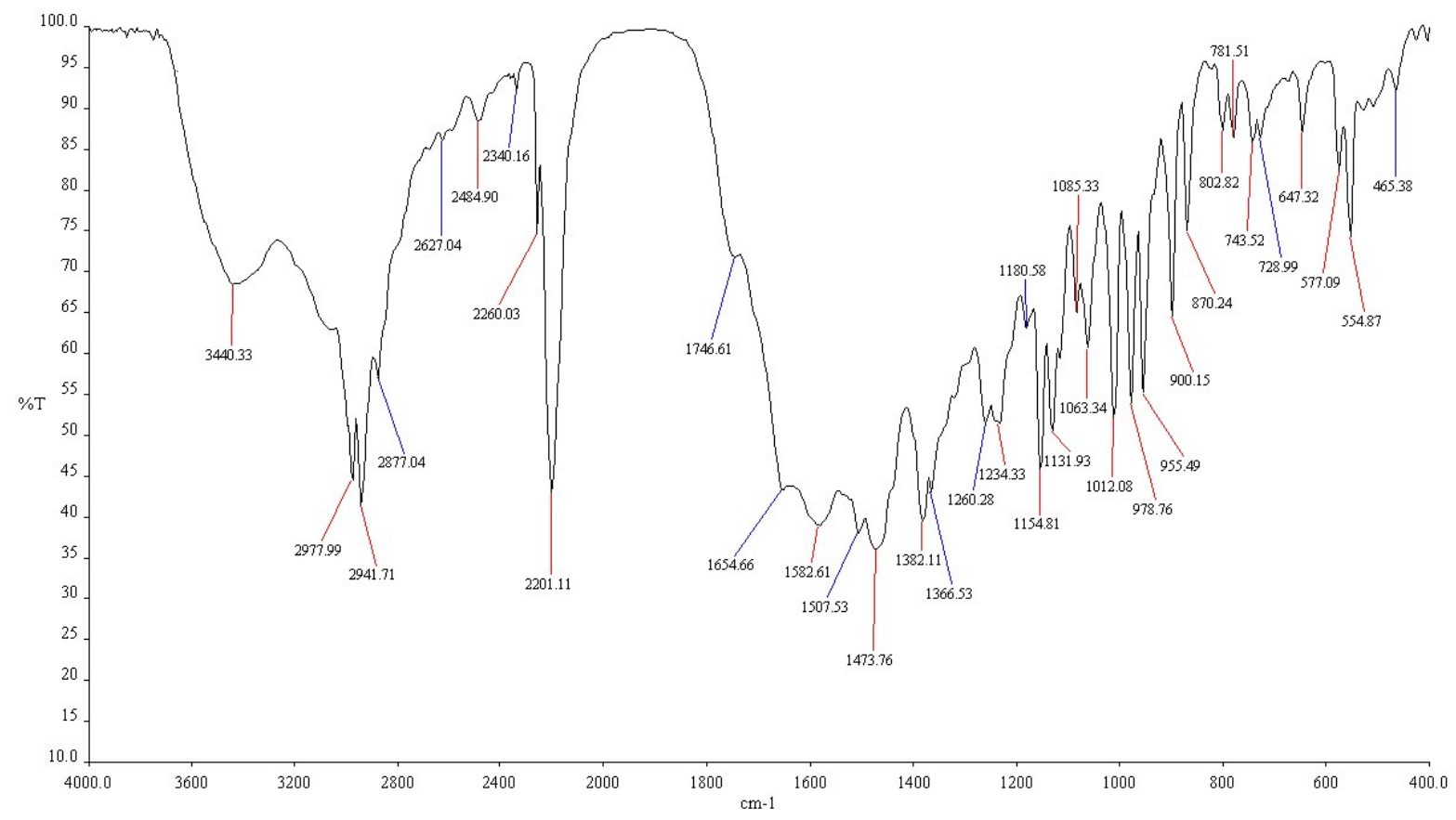

Figure 5 FTIR spectrum of TEMPO - TCNE

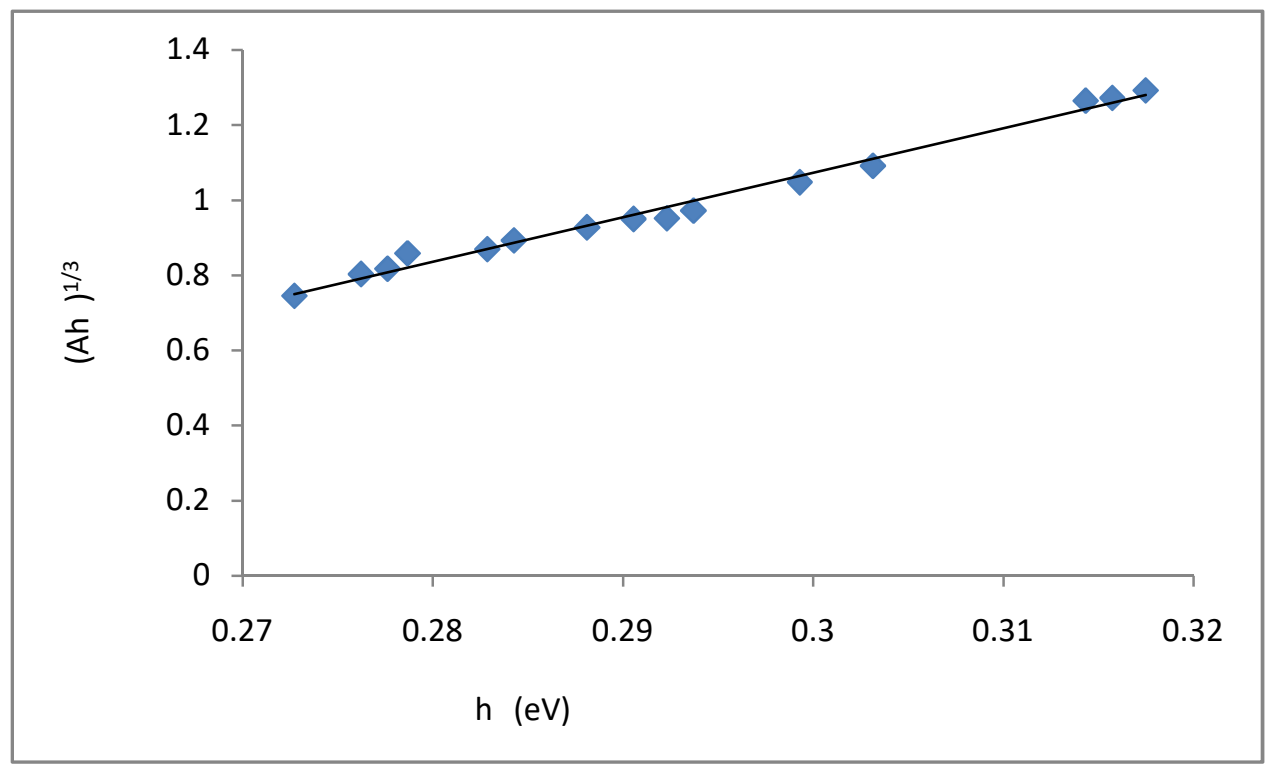

Figure $6 \mathrm{a}$ NT of TEMPO - TCNE 
International Journal of Trend in Scientific Research and Development, Volume 1(2), ISSN: 2456-6470 www.ijtsrd.com

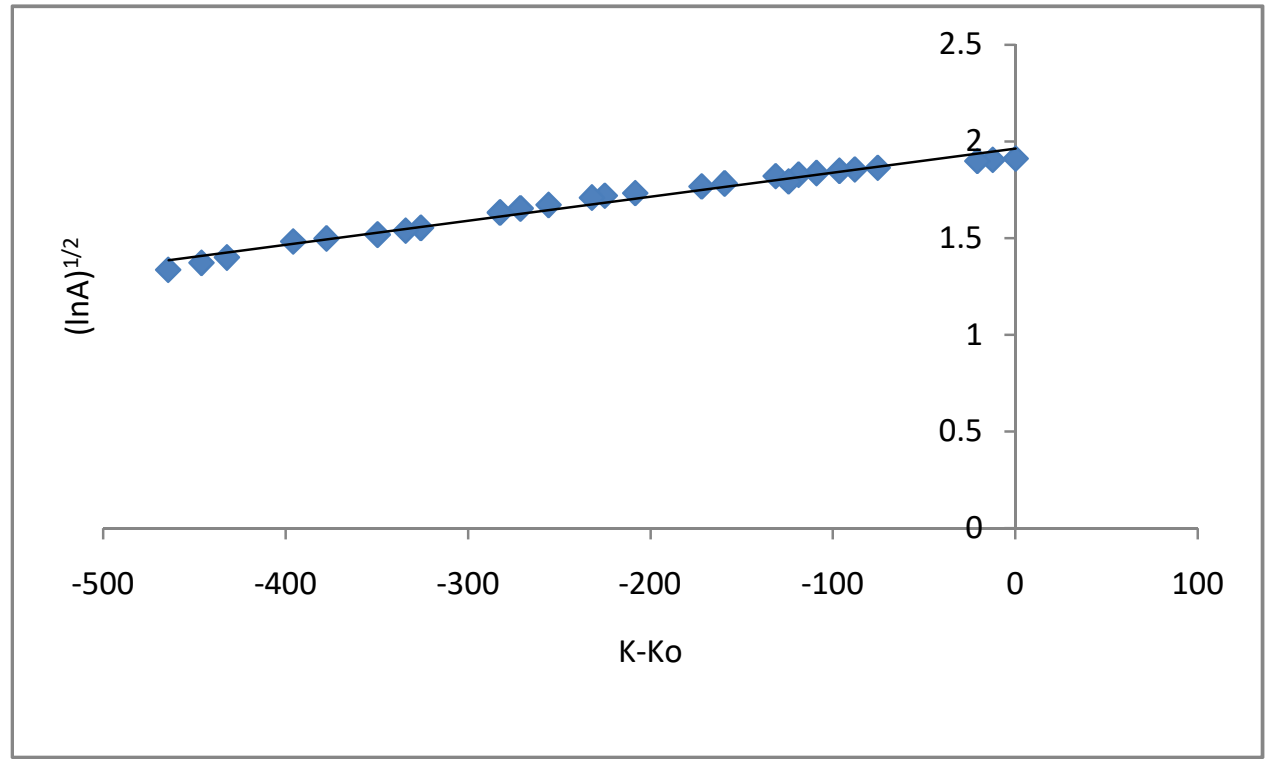

Figure 6b Assymmetric Gaussian of TEMPO - TCNE

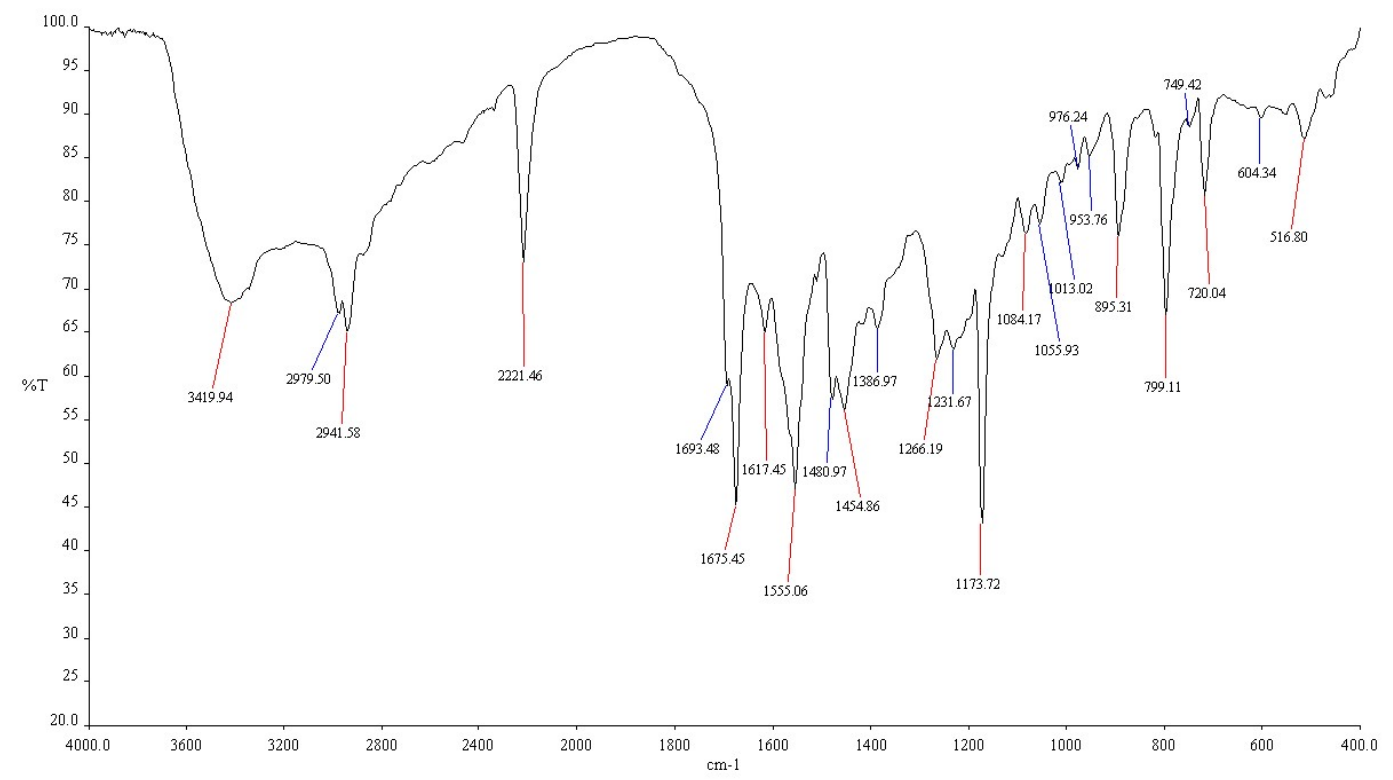

Figure 7 FTIR spectrum of TEMPO - DDQ 
International Journal of Trend in Scientific Research and Development, Volume 1(2), ISSN: 2456-6470 www.ijtsrd.com

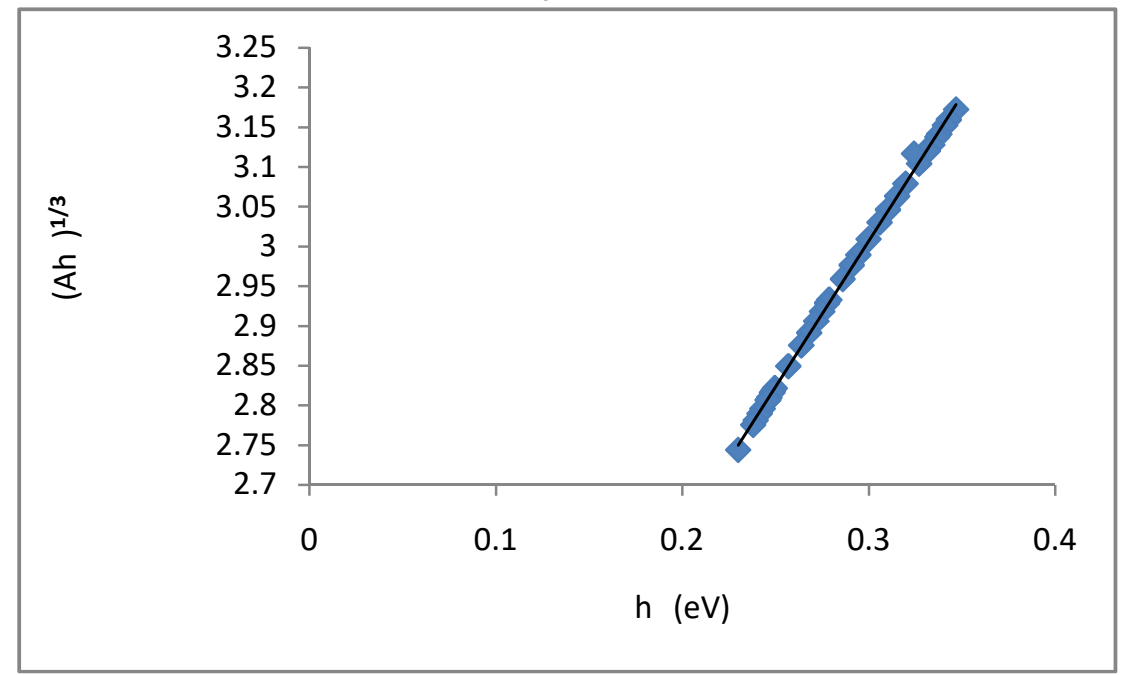

Figure 8a NT of TEMPO - DDQ

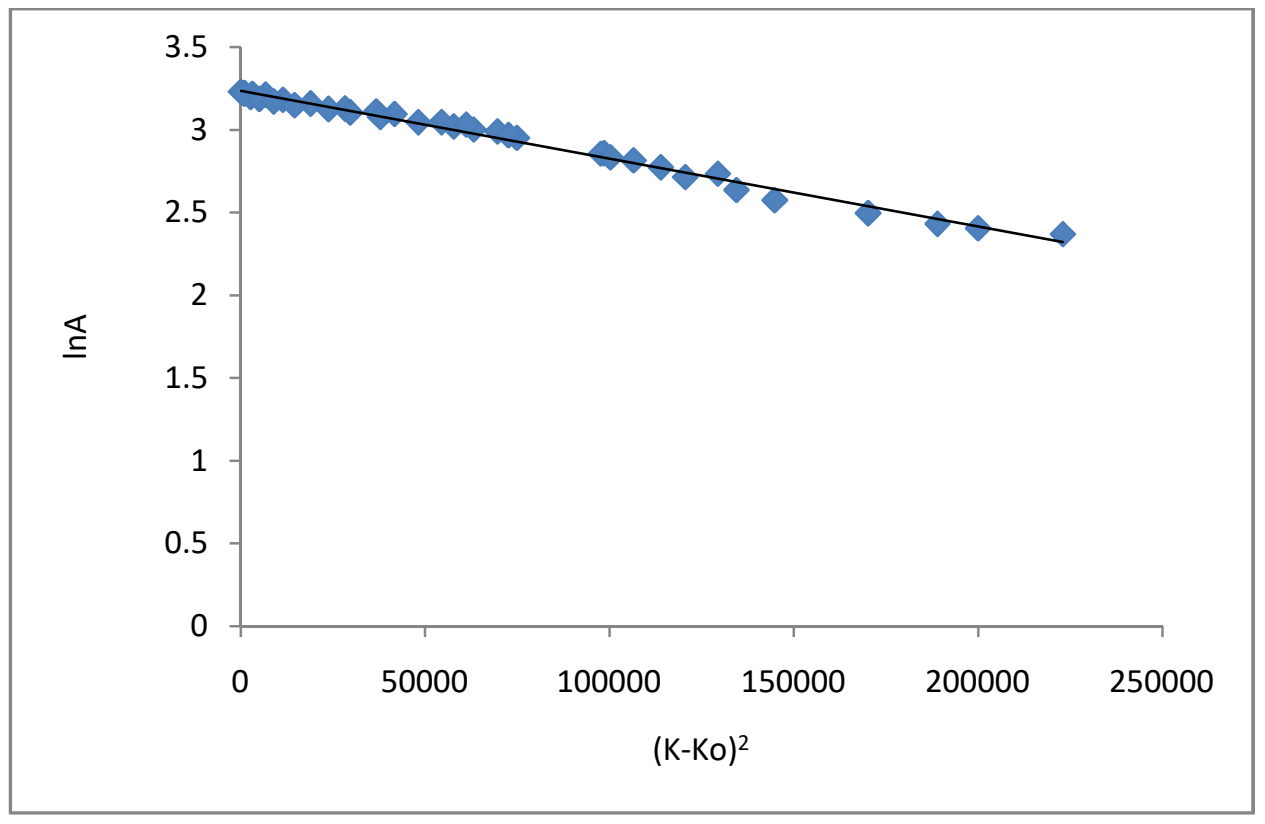

Figure 8b Symmetric Gaussian of TEMPO - DDQ 
International Journal of Trend in Scientific Research and Development, Volume 1(2), ISSN: 2456-6470 www.ijtsrd.com

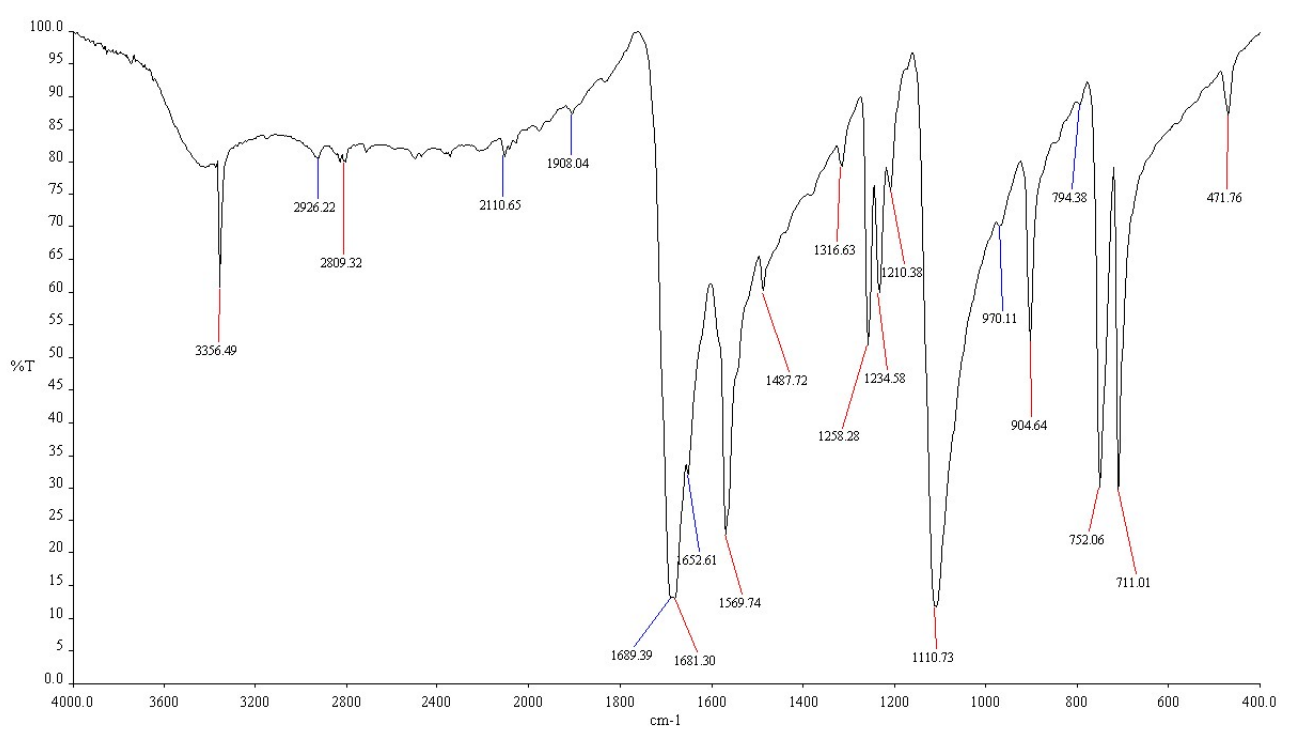

Figure 9 FTIR spectrum of TEMPO - Chloranil

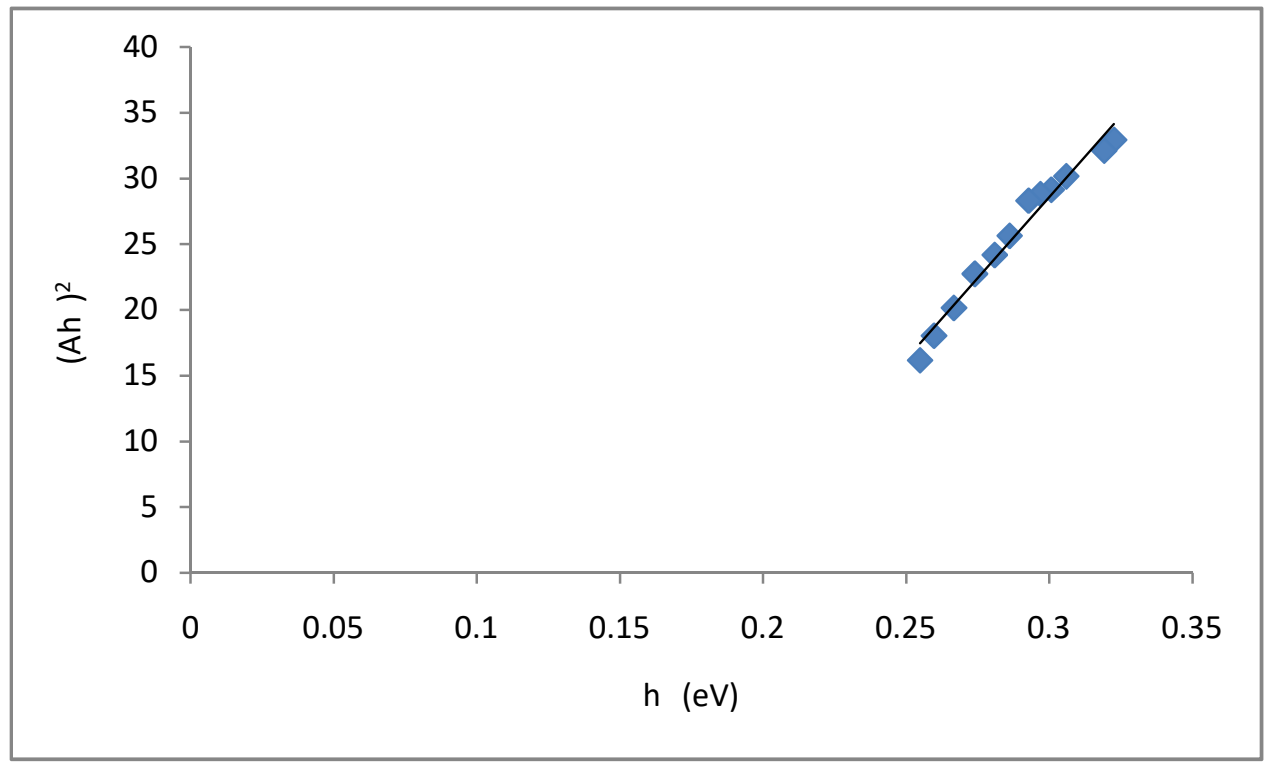

Figure 10 NT of TEMPO - Chloranil 
International Journal of Trend in Scientific Research and Development, Volume 1(2), ISSN: 2456-6470 www.ijtsrd.com

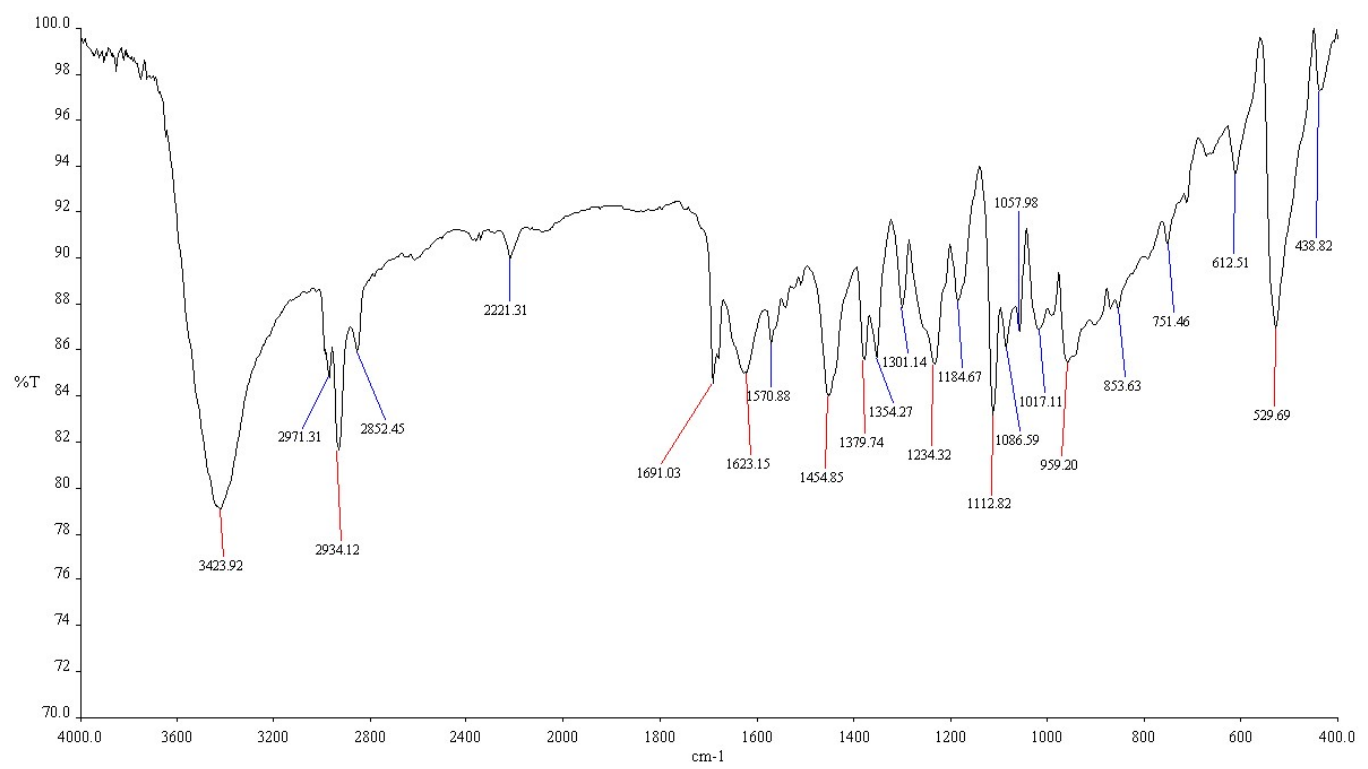

Figure 11 FTIR spectrum of TEMPO - IODINE

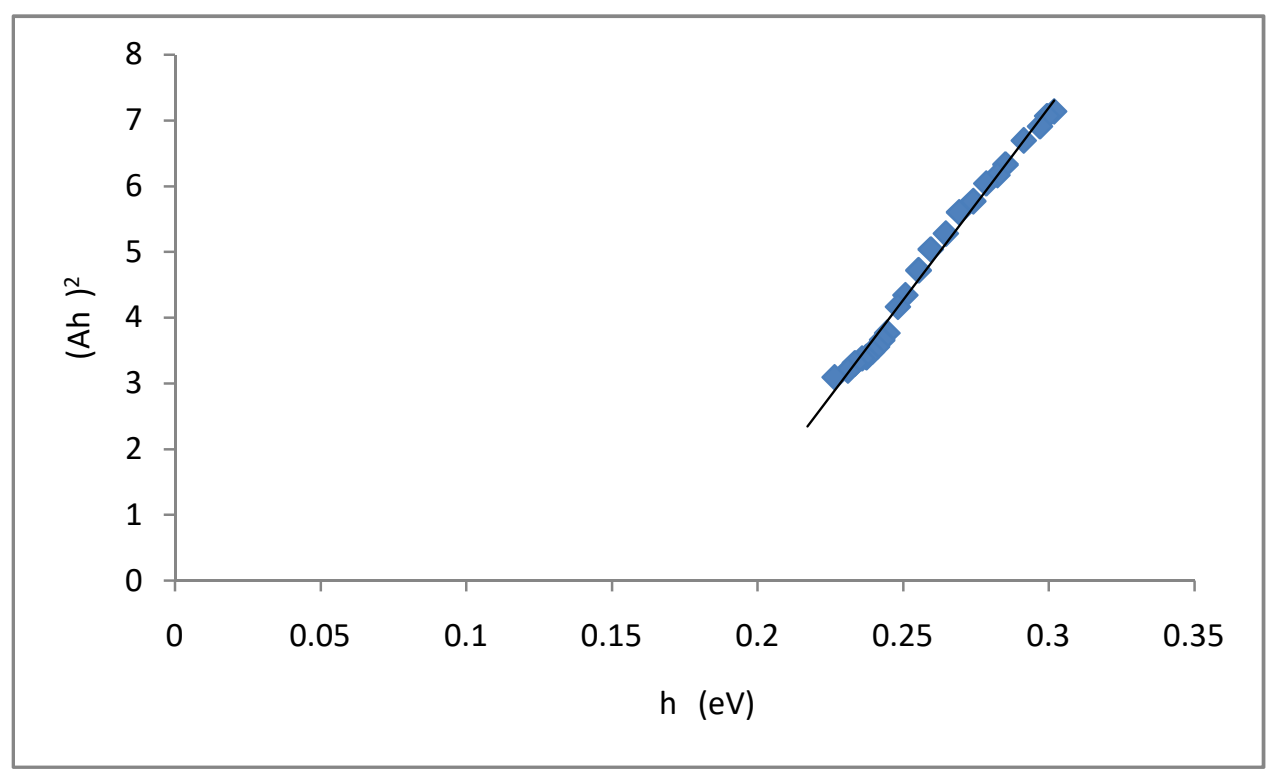

Figure 12a NT of TEMPO - IODINE 


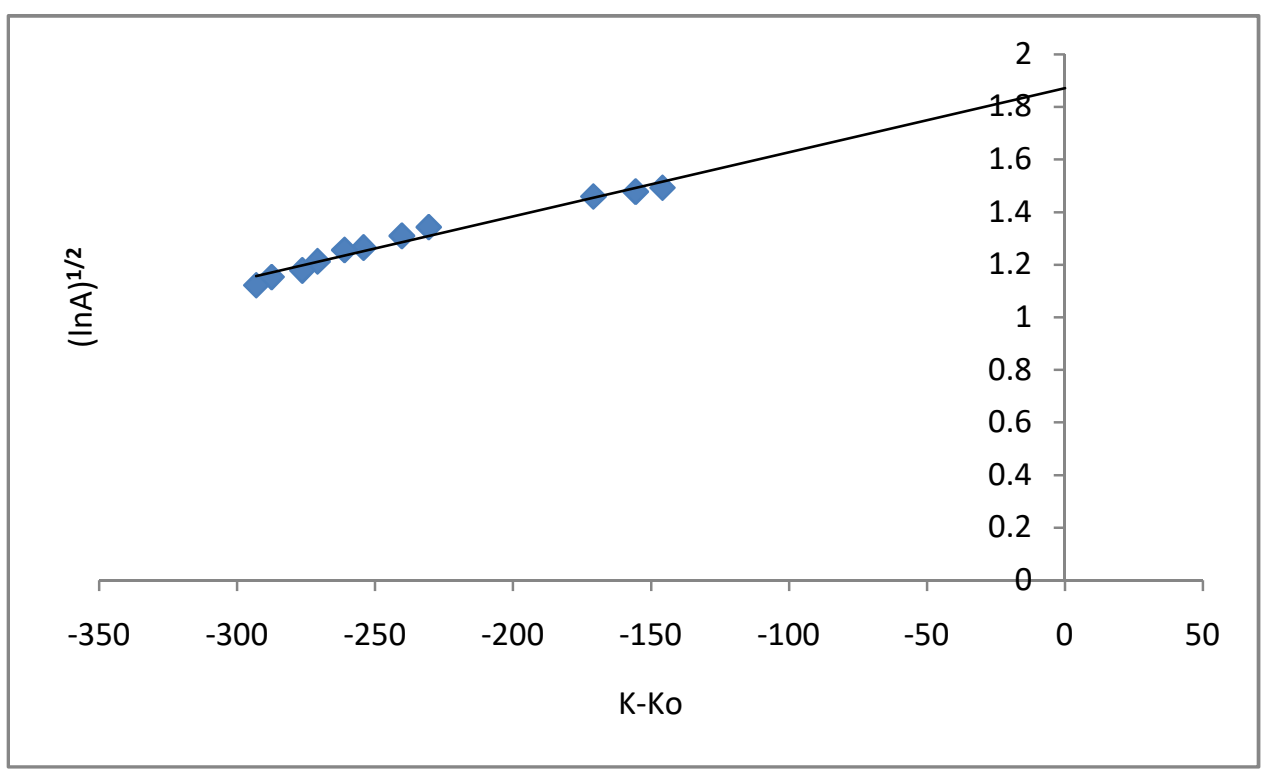

Figure 12b Assymmetric Gaussian of TEMPO - IODINE

\section{REFERENCES}

1. Shin'ichi Nakatsuji, ${ }^{\mathrm{a}}$ Atsushi Takai, ${ }^{\mathrm{a}}$ Kazuyoshi Nishikawa, ${ }^{\mathrm{b}}$ Yujio Morimoto, ${ }^{\mathrm{b}}$ Noritake Yasuoka, Kazuya Suzuki, ${ }^{\mathrm{c}}$ Toshiaki Enokid and Hiroyuki Anzai ${ }^{\mathrm{a}}$,Chem. Commun.,1997 27511

2. M. Tsujii, T. Sugumoto, H. Fujita, K.Ueda, and Y. Kai, Chem. Phys. Lett., 1996, 249, 304. 10

3. N. Mataga \& Cf.Y. Murata, Bull. Chem. Soc. Jpn., 1971, 44, 354.

4. C. Veyret ans A. Balise, Mol. Phys., 1973,25,873. 9

5. Cf. H.Iwamura Adv. Phys. Org. Chem., 1990, 26, 179; O. Kahn Molecular Magnetism, VCM, Weinheim, 1993; J. S. Miller and A. J. Epstein, Angew. Chem., Ed. Engl., 1994, 33, 385. 1-2

6. H.Sakurai, R.Kumai, A. Izuka and T. Sugawara,presented at The Vth International Conference on Molecular - based Magnets (ICMM 96), Osaka, July,1996, Abstract,p. 172, to be published. 